\title{
EXPANSIONS OF FIELDS BY ANGULAR FUNCTIONS
}

\author{
DAVID M. EVANS
}

\begin{abstract}
The notion of an angular function has been introduced by Zilber as one possible way of connecting non-commutative geometry with two 'counterexamples' from model theory: the nonclassical Zariski curves of Hrushovski and Zilber, and Poizat's field with green points. This article discusses some questions of Zilber relating to existentially closed structures in the class of algebraically closed fields with an angular function.

2000 Mathematics Subject Classification: $03 \mathrm{C} 45$.
\end{abstract}

\section{Introduction; Angular functions}

This article is motivated by some questions posed by Boris Zilber in [13]. In that paper, Zilber makes connections between noncommutative geometry and two 'counterexamples' in model theory: the non-classical Zariski curve of Hrushovski and Zilber from 10.1 of [4] (see Example 1.3 below), and Poizat's 'field with green points' from [9] (see Section 3.2 here). We will not attempt to describe these connections here: the reader should consult [13]. Instead we treat Zilber's questions purely in terms of their model-theoretic content.

We begin by saying what is meant by an angular function. For the moment, fix a natural number $N$, an algebraically closed field $F$ of characteristic zero and multiplicatively independent $\alpha, \beta \in F^{*}=$ $F \backslash\{0\}$. Let $\Gamma$ be the group of $N$-th roots of unity in $F$, and $\epsilon$ a primitive $N$-th root of unity. An angular function (with these data) is a function ang : $F^{*} \rightarrow \Gamma$ satisfying, for all $t \in F^{*}$ :

$$
\begin{aligned}
\operatorname{ang}(\epsilon t) & =\operatorname{ang}(t) \\
\operatorname{ang}(\beta t) & =\operatorname{ang}(t) \\
\operatorname{ang}(\alpha t) & =\epsilon \operatorname{ang}(t)
\end{aligned}
$$

In Question 2.4 of [13], Zilber asks:

Question 1.1. Consider a structure which is existentially closed in the class of structures $(F,+, \cdot, \alpha, \beta$, ang $)$ satisfying these equations. What is its model-theoretic status? Is it supersimple?

Date: 11 August 2006, Revised 4 September 2007. 
Before making more precise what our answer to this question is, we give some definitions and the examples which motivate the terminology and the question.

Note that associated to any angular function ang there are two definable subgroups of the multiplicative group of the field. The group of periods of ang is

$$
G=\left\{g \in F^{*}: \operatorname{ang}(g t)=\operatorname{ang}(t) \forall t \in F^{*}\right\} ;
$$

and the group of quasiperiods of ang is

$$
G^{+}=\left\{h \in F^{*}: \exists \gamma \in \Gamma \forall t \in F^{*} \operatorname{ang}(h t)=\gamma \operatorname{ang}(t)\right\} .
$$

So $\Gamma \leq G \leq G^{+}$and there is a definable homomorphism $\chi: G^{+} \rightarrow \Gamma$ with kernel $G$ (given by ang $(h t)=\chi(h) \operatorname{ang}(t)$ for $h \in G^{+}$and $t \in F^{*}$ ). Note that as defined above, $\chi$ is surjective, so the induced map $\bar{\chi}$ : $G^{+} / G \rightarrow \Gamma$ is an isomorphism.

Example 1.2. The following example from 2.3 of [13] provides the motivation for the terminology 'angular function.' Let $F=\mathbb{C}$, the complex numbers and $\epsilon=\exp (2 \pi i / N)$. For $k=0, \ldots, N-1$, let $P_{k}$ be the sector of the complex plane consisting of non-zero complex numbers $z$ with an $\operatorname{argument} \arg z$ in the range $2 \pi k / N \leq \arg z<2 \pi(k+1) / N$. We define ang $: \mathbb{C}^{*} \rightarrow \Gamma$ by, for $t \in \mathbb{C}^{*}$ :

$$
\operatorname{ang}(t)=\epsilon^{k} \Leftrightarrow t^{N} \in P_{k} .
$$

The group of periods of ang is $G=\mathbb{R}^{>0} \Gamma$ and the group of quasiperiods is $G^{+}=\mathbb{R}^{>0}\left\langle\epsilon_{1}\right\rangle$ where $\epsilon_{1}=\exp \left(2 \pi i / N^{2}\right)$.

It is clear that $(\mathbb{C},+, \cdot$, ang) has the strict order property (consider additive translates of the definable subset $P_{0}$ ).

Example 1.3. We describe briefly, following Section 1 of [13], how to obtain an example of a non-classical Zariski curve from a suitable angular function. Given $(F ;+, \cdot, \alpha, \beta$, ang $)$ satisfying the above equations, (and where $\langle\alpha, \beta\rangle$ is free abelian of rank 2) define $U, V: F^{*} \rightarrow F^{*}$ by

$$
\begin{aligned}
U(t) & =\alpha t \\
V(t) & =\beta \operatorname{ang}(t) t .
\end{aligned}
$$

These are definable permutations of $F^{*}$ and

$$
V U(t)=\epsilon U V(t) .
$$

Let $T$ denote the set $F$ with only the structure given by the definable permutations $U, V$ and $p: T \rightarrow F$ given by $p(t)=t^{N}$. Then the structure $((T ; U, V),(F ;+, \cdot), p: T \rightarrow F)$ is a finite cover of $(F ;+, \cdot)$ which is interpretable in $(F ;+, \cdot$, ang $)$, but not in $(F ;+, \cdot)$. More detatils can be found in Section 1 of [13] and Section 10 of [4].

We now return to Question 1.1. The intention is to add a generic angular function, having prescribed in advance the groups of periods and 
quasiperiods (rather than also having an existential closure condition on, say, the group of periods). Thus we make the following definitions.

Definition 1.4. Let $L_{0}$ be a first-order language which contains the language of rings $(+,-, \cdot, 0,1)$ and additional unary predicates $\mathbf{F}, \boldsymbol{\Gamma}, \mathcal{G}$ and $\mathcal{G}^{+}$, and a unary function symbol $\chi$. Let $F$ be an algebraically closed field of characteristic 0 , and $\Gamma \leq G \leq G^{+} \leq F^{*}$ subgroups of the multiplicative group of $F$. Suppose $\chi: G^{+} \rightarrow \Gamma$ is a surjective homomorphism with kernel $G$. We consider this as an $L_{0^{-}}$-structure, where $\mathbf{F}$ is interpreted as $F, \boldsymbol{\Gamma}$ as $\Gamma, \mathcal{G}$ as $G, \mathcal{G}^{+}$as $G^{+}$and $\chi$ as $\chi$. Let $T_{0}=T h\left(F ;+,-, \cdot, 0,1, \Gamma, G, G^{+}, \chi, \ldots\right)$ be the $L_{0}$-theory of this structure. Without loss, we shall assume that $L_{0}$ has been extended so that $T_{0}$ is model-complete. Note that if $\Gamma$ is finite, then $G^{+}$and $\chi$ are definable (with parameters) from $G$ in the multiplicative group $F^{*}$.

Now let $L_{A}$ be the expansion of $L_{0}$ by an extra unary function symbol $A$. We define $T_{A}$ to be the theory axiomatized by $T_{0}$ and axioms:

(i) $(A(0)=0) \wedge(\forall t)((t \neq 0) \rightarrow \Gamma(A(t)))$;

(ii) $(\forall t)(\forall g)(\mathcal{G}(g) \rightarrow A(g \cdot t)=A(t))$;

(iii) $(\forall t)(\forall h)\left(\mathcal{G}^{+}(h) \wedge(t \neq 0) \rightarrow A(h \cdot t)=\chi(h) \cdot A(t)\right)$

Thus in a model $M$ of $T_{A}$, the subgroups $\mathcal{G}(M)$ and $\mathcal{G}^{+}(M)$ are contained in the periods and quasiperiods of the angular function $A^{M}$ and it is easy to show that if $M$ is an existentially closed model of $T_{A}$, then they are exactly the periods and quasiperiods ( a priori it is not immediately obvious that $T_{A}$ is even consistent, but this will become clear from Lemma 2.1). Question 1.1 can then be seen as asking whether the class of existentially closed models of $T_{A}$ is axiomatizable, and if so whether completions of its theory are supersimple. In Section 2 we prove:

Theorem 1.5. (1) If $T_{0}$ eliminates the quantifier $\exists^{\infty}$ in the sorts $\mathbf{F}, \mathbf{F} / \mathcal{G}$ and $\mathbf{F} / \mathcal{G}^{+}$then $T_{A}$ has a model companion $T_{A}^{*}$.

(2) If additionally $T_{0}$ is simple and $\Gamma$ is finite then all completions of $T_{A}^{*}$ are simple (and in the same simplicity class as $T_{0}$ ).

By the parenthetic remark at the end of (2), we mean, for example, that if $T_{0}$ is supersimple of $S U$-rank $\kappa$, then so is any completion of $T_{A}^{*}$. If $\Gamma$ is non-trivial, then $T_{A}^{*}$ will not be stable.

Essentially both parts of the theorem follow quickly from known results. As we shall see, the first follows from an old theorem of Winkler [10], and the second follows from results of Nübling in [5]. Of course, for the result to be of any relevance to Zilber's question we need to identify suitable $T_{0}$ which satisfy its hypotheses.

We consider two situations where the model theory of an algebraically closed field of characteristic zero expanded by a predicate for a subgroup of the multiplicative group is understood. In the first case, the 
subgroup is in the divisible hull of a finitely generated group (- structures of Lang type). In the second case, we consider structures constructed via a Hrushovski predimension as in Poizat's 'field with green points' from [9] (- structures of Poizat type). The first case is relevant to Section 2 in Zilber's paper [13] and the second case is relevant to Sections 3 and 4 there.

In both cases we are dealing with superstable structures (of infinite $U$-rank). Both cases have weak nfcp, that is, they eliminate $\exists^{\infty}$ in all (real and imaginary) sorts. For structures of Lang type, this is folk-lore; for structures of Poizat type we give a proof using belles paires.

In Section 4 we show how to put a probability measure on definable sets in models of $T_{A}^{*}$, giving one possible answer to the problem at the end of Section 2 of [13].

\section{WinkleR's TheOrem AND The PROOF OF TheOREM 1.5}

Throughout this section we use the notation of Definition 1.4. Suppose we have a model $M$ of $T_{A}$. If the model is understood from the context, we will abuse notation and write $\mathcal{G}$ instead of $\mathcal{G}(M)$ etc.

Rather than considering the angular function $A: \mathbf{F} \rightarrow \boldsymbol{\Gamma}$ we consider a section $s: \mathbf{F}^{*} / \mathcal{G}^{+} \rightarrow \mathbf{F}^{*} / \mathcal{G}$ of the natural map $\nu: \mathbf{F}^{*} / \mathcal{G} \rightarrow \mathbf{F}^{*} / \mathcal{G}^{+}$ (given by $\nu(x \mathcal{G})=x \mathcal{G}^{+}$) which is interdefinable with $A$. More formally, suppose $L_{0}$ has been expanded to include the part of $L_{0}^{e q}$ involving the sorts $\mathbf{F}^{*} / \mathcal{G}^{+}$and $\mathbf{F}^{*} / \mathcal{G}$. Expand $L_{0}$ further to a language $L_{s}$ which has an extra unary function symbol $s$ between these sorts. Let $T_{s}$ be the $L_{s}$-theory axiomatized by $T_{0}$ and the axiom:

$$
\left(\forall y \in \mathbf{F}^{*} / \mathcal{G}^{+}\right)\left(s(y) \mathcal{G}^{+}=y\right)
$$

saying that $s$ is a section of the $L_{0}$-definable map $\nu$.

Lemma 2.1. There is a definable correspondence between the models of $T_{A}$ and the models of $T_{s}$ which preserves the property of existential closure. Thus $T_{A}$ has a model companion (respectively, model completion) if and only if $T_{s}$ does.

Proof: The correspondence is given by the equation:

$$
A(t)=\bar{\chi}\left(s\left(t \mathcal{G}^{+}\right)^{-1} t \mathcal{G}\right) .
$$

Indeed, given a section $s$ of $\nu$, then $s\left(t \mathcal{G}^{+}\right)^{-1} t \mathcal{G} \in \mathcal{G}^{+} / \mathcal{G}$ and $\bar{\chi}$ maps this into $\boldsymbol{\Gamma}$. So $A$ given by this equation is certainly a map from $\mathbf{F}^{*}$ to $\Gamma$ and it is easy to check that it satisfies the axioms in Definition 1.4. Conversely given an angular function $A$, the above equation determines a function $s: \mathbf{F}^{*} / \mathcal{G}^{+} \rightarrow \mathbf{F}^{*} / \mathcal{G}$ given by:

$$
s\left(t \mathcal{G}^{+}\right)=t \mathcal{G}\left(\bar{\chi}^{-1}(A(t))\right)^{-1},
$$

because $\bar{\chi}$ is bijective, and one checks easily that this is a section of $\nu$.

The above correspondence is given by a quantifier-free formula, so it translates existentially closed structures in one class into existentially 
closed structures in the other. This proves the statement about the existence of model companions. For the statement about model completions, observe that $T_{A}$ has the amalgamation property iff $T_{s}$ has the amalgamation property.

By the above lemma it will suffice to prove Theorem 1.5 for the theory $T_{s}$. The advantage of this is that $s$ only has a simple axiom to satisfy: that of being a section of a certain definable map. This is exactly the situation in which we can apply a result of Winkler from the 1970's. In the following, $L$ is any first-order language and $T$ any $L$-theory.

Definition 2.2. We say that $T$ eliminates the quantifier 'there exist infinitely many' (or eliminates $\exists^{\infty}$, or is algebraically bounded) if for all $L$-formulas $\phi(x, \bar{y})$ there is a natural number $N_{\phi}$ with the property that for all models $M$ of $T$ and $\bar{a}$ in $M$, if $\phi[M, \bar{a}]$ has more than $N_{\phi}$ elements, then it is infinite.

Definition 2.3. Suppose $\phi(x, \bar{y})$ is an $L$-formula, where $\bar{y}$ is an $n$-tuple of variables. Let $L^{+}$be the expansion of $L$ by a new $n$-ary function symbol $\sigma$. The $L^{+}$theory $T^{+}$is axiomatized by $T$ together with the new axiom:

$$
(\forall \bar{y})((\exists x) \phi(x, \bar{y}) \rightarrow \phi(\sigma(\bar{y}), \bar{y})) .
$$

We refer to $T^{+}$as a Skolem expansion of $T$.

So of course this says that $\sigma$ is a Skolem function for the formula $\phi(x, \bar{y})$. Strictly speaking, $\sigma$ should be defined for all $n$-tuples, even if they do not satisfy $(\exists x) \phi(x, \bar{y})$. We can avoid this issue by including a sort for the definable set $(\exists x) \phi(x, \bar{y})$ and only defining $\sigma$ on this sort. Alternatively we can define $\sigma(\bar{y})$ to be some fixed $\emptyset$-definable element.

Theorem 2.4 (P. Winkler, Theorem 2 of [10]). Suppose $T$ is a modelcomplete $L$-theory which eliminates the quantifier $\exists^{\infty}$. Then any Skolem expansion $T^{+}$of $T$ has a model completion $\left(T^{+}\right)^{*}$.

Proof of Theorem 1.5: (1) The theory $T_{s}$ is the Skolem expansion of $T_{0}$ with respect to the formula $\phi(x, y)$ :

$$
(x \in \mathbf{F} / \mathcal{G}) \wedge\left(y \in \mathbf{F} / \mathcal{G}^{+}\right) \wedge\left(x \mathcal{G}^{+}=y\right) .
$$

The assumptions on eliminating $\exists^{\infty}$ in the indicated sorts means that we can apply Winkler's Theorem to deduce that $T_{s}$ has a model completion $T_{s}^{*}$. So by Lemma 2.1 there is a model completion $T_{A}^{*}$ of $T_{A}$.

(2) If $\Gamma$ is finite, then $\Gamma$ is finite in all models of $T_{0}$ and the map $\nu$ is $|\Gamma|$-to-1. So the Skolem expansion $T_{s}$ is an algebraic Skolem expansion (in the terminology of [5]) and the results in Section 1 of [5] give immediately that if $T_{0}$ is simple then so is any completion of $T_{s}^{*}$ (and in the same simplicity class). By interdefinability of $T_{A}$ and $T_{s}$, the same is true of $T_{A}^{*}$. 
Remarks 2.5. The result in [10] is more general than is stated in Theorem 2.4: one has a model completion for the expansion by an arbitrary number of Skolem functions. By Theorem 4 of [10] there is also a converse to Theorem 2.4: if there is a model companion for $T^{+}$then $T^{+}$eliminates $\exists^{\infty}$. By combining these results, one obtains ([10], Corollary 1) that the model completion $\left(T^{+}\right)^{*}$ in Theorem 2.4 eliminates $\exists^{\infty}$.

The proof of Theorem 2.4 gives an axiomatization of $T_{s}^{*}$. As well as $T_{s}$ we have axioms of the form:

$$
\forall \bar{x}\left(\left(\exists^{\infty} \bar{y}\right) \alpha(\bar{x}, \bar{y}) \rightarrow(\exists \bar{y})(\alpha(\bar{x}, \bar{y}) \wedge \beta(\bar{x}, \bar{y}))\right)
$$

where

(i) $\alpha$ is an $L_{0}$-formula and $\beta$ is a conjunction of formulas of the form ' $s\left(v_{1}\right)=v_{2}$ ' where $v_{1}, v_{2}$ are amongst the variables in $\bar{x}, \bar{y}$, not both in $\bar{x}$ (and no variable appearing as $v_{1}$ is in more than one such conjunct).

(ii) If $i \neq j$ then $y_{i} \neq y_{j}$ is a conjunct of $\alpha$ and if ' $s\left(v_{1}\right)=v_{2}$ ' is a conjunct of $\beta$, then $\phi\left(v_{2}, v_{1}\right)$ is a conjunct of $\alpha$.

It should be noted that ' $\exists^{\infty} \bar{y} \ldots$.' here means 'there exist infinitely many $\bar{y}$ which are different in each coordinate' (see the definition in Section 1 of [10]).

Remarks 2.6. We note that if, in Theorem 1.5 the group $\Gamma$ is not finite (and $\mathbf{F}^{*} / \mathcal{G}^{+}$is infinite), then $T_{s}^{*}$ and $T_{A}^{*}$ cannot be simple. The argument is essentially that of Lemma 3.1 of [5]. In fact, the formula $\theta(x ; y, z)$ given by $A(x \cdot y)=z$ has the tree property (with respect to $\left.T_{s}^{*}\right)$. In some model of $T_{s}^{*}$ take $\left(a_{i}: i<\omega\right)$ in $\mathbf{F}^{*}$ lying in different $\mathcal{G}^{+}$-cosets, and distinct elements $\left(g_{j}: j<\omega\right)$ of $\boldsymbol{\Gamma}$. For $\eta \in \omega^{<\omega}$ with domain $n>0$ consider the parameters $c_{\eta}=\left(a_{n}, g_{\eta(n-1)}\right)$. Clearly $\left.\left\{\theta\left(x ; c_{\left(\eta^{\wedge} j\right.}\right)\right): j<\omega\right\}$ is pairwise inconsistent for each $\eta \in \omega^{\omega}$, and using the above axioms for $T_{s}^{*}$ one shows that for $\zeta \in \omega^{\omega}$ the set $\left\{\theta\left(x ; c_{\zeta \mid n}\right): n<\omega\right\}$ is consistent.

\section{WEAK NFCP}

Suppose $L$ is a first-order language and $T$ a complete $L$-theory. Then by a theorem of Shelah, $T$ has nfcp ('does not have the finite cover property') if and only if it is stable and eliminates the quantifier $\exists^{\infty}$ in all (real and imaginary) sorts. The latter property (for arbitrary $T$ ) is sometimes referred to as weak nfcp. In this section we discuss two types of stable theories $T_{0}$ to which we would like to apply Theorem 1.5 , and show that they have weak nfcp.

3.1. Structures of Lang type. Recall that an abelian group is said to be of finite rank if it is in the divisible hull of a finitely generated subgroup. By a structure of Lang type we mean an algebraically closed 
field of characteristic zero expanded by a predicate $\mathcal{G}$ for a finite rank subgroup $G$ of some semiabelian variety (see [7], where the use of this terminology is different, but consistent with ours). We shall be interested in the particular case where $G$ is a finite rank subgroup of the multiplicative group (though it should be noted that the following also holds in the more general context).

Theorem 3.1. Suppose $F$ is an algebraically closed field of characteristic 0 and infinite transcendence rank and $G$ is a finite rank subgroup of the multiplicative group $F^{*}$. Consider this as an L-structure where $L$ is the language of rings expanded by a unary predicate $\mathcal{G}$ for the subgroup $G$. Then:

(i) The induced structure on $\mathcal{G}$ is that of a stable one-based group and if $G$ is $\kappa$-stable, then so is $T=T h(F ;+, \cdot, 0,1, \mathcal{G})$.

(ii) $T$ has $n f c p$.

Proof: Part (i) is from [7], particularly Proposition 2.6.

For (ii) one can easily adapt the proof of Proposition 2.6 of [7] to show that $T$ is non-multidimensional. It follows (for example by Remark 8.2.13 of [6]) that $T$ has nfcp. Alternatively, by (i) the induced structure on $\mathcal{G}$ is that of a one-based group and so has nfcp. Moreover, $G$ is small in $F$ (in the terminology of [1]), so one can apply Proposition 5.7 of [1] to obtain that $T$ has nfcp.

We summarise our answer to Zilber's question (Question 1.1) as follows, using the notation $\left(L_{0}, T_{0}, L_{A}, T_{A}\right)$ from Definition 1.4.

Theorem 3.2. Suppose $F$ is an algebraically closed field of characteristic zero of infinite transcendence rank and $\alpha, \beta \in F$ are multiplicatively independent. Let $N$ be a natural number, $\epsilon$ a primitive $N$-th root of 1 and $\Gamma=\langle\epsilon\rangle ; G=\left\langle\alpha^{N}, \beta, \epsilon\right\rangle ;$ and $G^{+}=\langle\alpha, \beta, \epsilon\rangle$. Define $\chi: G^{+} \rightarrow \Gamma$ to have kernel $G$ and $\chi(\alpha)=\epsilon$. Then:

(i) $T_{0}=\operatorname{Th}\left(F ;+,-, \cdot, 0,1, \Gamma, G, G^{+}, \chi\right)$ is superstable of Lascar rank $\omega$ and has nfcp.

(ii) $T_{A}$ has a model completion $T_{A}^{*}$ and all completions of this are supersimple of $S U$-rank $\omega$.

Proof: (i) All of the structure is interpretable in $(F, G, \alpha, \beta, \epsilon)$ and the required properties of this are given by Theorem 3.1 and the proof of Proposition 2.6 in [7].

(ii) This follows from part (i) and Theorem 1.5.

Of course, in a model of $T_{A}$ as above the function $A$ is an angular function, that is, it satisfies the properties (1)-(3) in the Introduction.

By Theorem 10 of [10], any one-cardinal model of $T_{0}$ can be expanded to a model of $T_{A}^{*}$. In particular, there is a countable model of $T_{A}^{*}$ in which $\mathcal{G}=G$. It is natural to ask whether there is a model of $T_{A}^{*}$ in which the field is the complex numbers and the group of periods is 
precisely $G$. To do this it would be enough to construct an e.c. model of $T_{A}$ (or of $T_{s}$ ) of cardinality continuum in which $\mathcal{G}=G$. This cannot be an entirely straightforward union-of-a-chain argument, as the following example shows.

Let $M_{0}$ be a model of $T_{0}$, let $c \in \mathbf{F}\left(M_{0}\right)$ be algebraically independent from $\mathcal{G}\left(M_{0}\right)$ and $\psi(x, y, c)$ be the q.f. formula $x \in \mathcal{G} \wedge y=x+c$. Then $M_{0}$ can be expanded to a model $M$ of $T_{s}$ in which $s(\nu(y \mathcal{G})) \neq y \mathcal{G}$ for all $y \in c+\mathcal{G}$. On the other hand, $c+\mathcal{G}\left(M_{0}\right)$ meets infinitely many (multiplicative) cosets modulo $\mathcal{G}$, so there is an elementary extension $N_{0}$ of $M_{0}$ and $b \in \mathcal{G}\left(N_{0}\right)$ such that $(b+c) \mathcal{G}\left(N_{0}\right) \notin \mathbf{F}\left(M_{0}\right) / \mathcal{G}\left(N_{0}\right)$. Thus we can expand $N_{0}$ to a model $N$ of $T_{s}$ so that $M$ is a submodel of $N$ and $\psi(x, y, c) \wedge s(\nu(y \mathcal{G}))=y \mathcal{G}$ has a solution $(b, b+c)$ in $N$. It follows that any e.c. model $M_{1}$ of $T_{s}$ which contains $M$ has to have $\mathcal{G}\left(M_{1}\right)>\mathcal{G}(M)$.

3.2. Poizat's field with green points. We wish to consider Poizat's 'field with green points' $(F, G(F))$ from [9]. This is a structure in a language $L$ for fields with an extra unary predicate $G$. The field $F$ is algebraically closed of characteristic zero and the subset $G(F)$ (- the 'green points') is a torsion free divisible subgroup of the multiplicative group $F^{\times}$. For every algebraically closed subfield $A$ of finite transcendence degree we have the predimension inequality

$$
\delta(A)=2 \operatorname{trdeg}(A)-\operatorname{rk}_{\mathbb{Q}} G(A) \geq 0 .
$$

Moreover $(F, G(F))$ is 'rich' : the familiar existential closure condition with respect to self-sufficient embeddings. The first-order axiomatization of $T_{G}=T h(F, G(F))$ is described in Section 3 of [9].

The main result of this Section is:

Theorem 3.3. $T_{G}$ has nfcp.

It should be noted that we will not use $T_{G}$ when applying Theorem 1.5. Instead, we will use variations on Poizat's original construction (see Section 3.3). The axiomatisation of these variations and the proofs of nfcp are similar to those for $T_{G}$, and it therefore seems reasonable to present the proof of nfcp for the original 'field with green points.'

The proof we give of Theorem 3.3 uses Poizat's technology of belles paires from [8] and we begin by briefly recalling this.

Let $P$ be a new unary predicate symbol and $L^{P}$ the language obtained by adjoining $P$ to $L$. We consider a pair $M \preceq N$ of models of $T_{G}$ as an $L^{P}$-structure by interpreting $P$ as the subset $M$. We refer to this as a belle paire if $M$ is $\omega^{+}$-saturated (in the $L$-sense) and for every finite subset of $N$, every 1-type (in the $L$-sense) over $M \cup A$ is realised in $N$. Let $T_{G}^{P}$ denote the $L^{P}$-theory of all belles paires. As 
$T_{G}$ is stable, $T_{G}^{P}$ is complete ([8], Théorème 4) and $T_{G}$ has nfcp iff every $\omega^{+}$-saturated (in the $L^{P}$-sense) model of $T_{G}^{P}$ is a belle paire ([8], Théorème 6).

Henceforth, let $M \preceq N$ be an $\omega^{+}$-saturated model of $T_{G}^{P}$. We need to show that this is a belle paire, and of course, the issue is the relative $\omega$ saturation of $N$ over $M$. Let cl denote self-sufficient closure in $N$, and $\mathrm{acl}_{f}$ denote field-theoretic algebraic closure. Let $d$ be the dimension function associated with the predimension $\delta$.

Let $A$ be a finite subset of $N$ and $p(x)$ a 1-type (in the $L$-sense) over $M \cup A$. Let $M_{1}=\operatorname{cl}_{N}(M \cup A)$. We want to show that $p$ is realised in $N$. Suppose it is realised by $c$ in the elementary extension $N_{1}$ of $N$. We may assume that $M \preceq N_{1}$ is a belle paire, so $(M, N) \preceq_{L^{P}}\left(M, N_{1}\right)$. Now, $M_{2}=\operatorname{cl}_{N_{1}}(M \cup A \cup\{c\})$ is of finite transcendence degree over $M_{1}$ ([9], Corollaire 1.4), so we may assume that $M_{1} \leq M_{2}$ is a minimal extension: for every algebraically closed $C$ with $M_{1} \subset C \subseteq M_{2}$, we have $\operatorname{cl}(C)=M_{2}$. There are three possible cases to consider, depending on $d\left(c / M_{1}\right)$ (computed in $N_{1}$ ).

Case 1. (White generic) $d\left(c / M_{1}\right)=2$. So $c \notin M_{1} ; G\left(M_{1}\right)=G\left(M_{2}\right)$ and $M_{2}=\operatorname{acl}_{f}\left(M_{1} c\right)$.

Case 2. (Green generic) $d\left(c / M_{1}\right)=1$. So $c \notin M_{1} ; c$ is green; $G\left(M_{1}\right)$ is in the divisible hull of the subgroup generated by $G(M)$ and $c ; \operatorname{acl}_{f}\left(M_{1} c\right)=M_{2}$.

Case 3. $d\left(c / M_{1}\right)=0$.

Claim 1. For any natural number $n$ there exist $g_{1}, \ldots, g_{n} \in G(N)$, algebraically independent over $M$, with $d\left(g_{1}, \ldots, g_{n} / M\right)=n$.

So the claim is that $N$ contains a Morley sequence $g_{1}, \ldots, g_{n}$ of length $n$ for the green generic over $M$ : the $g_{i}$ should be green points, algebraically independent over $M$, and $\operatorname{acl}_{f}\left(M g_{1} \ldots g_{n}\right)$ should selfsufficient in $N$, with its $G$-part $\mathbb{Q}$-dependent on $G(M)$ and $g_{1}, \ldots, g_{n}$. We show that there is a set $\Phi\left(x_{1}, \ldots, x_{n}\right)$ of $L^{P}$-formulas (without parameters) which is equivalent to the $L(M)$-type of such a sequence. The claim then follows, because of the $\omega^{+}$-saturation of the pair $(M, N)$.

The formulas should express that:

- the $g_{i}$ are in $G$ and algebraically independent over the $P$-part $M$

- for any $s \geq 0$, if $e_{1}, \ldots, e_{2 s+1}$ are green and have transcendence degree at most $s$ over $M \bar{g}$, then they are multiplicatively dependent over $G(M) \bar{g}$

Note that the case $s=0$ is equivalent to the $G$-part of $\operatorname{acl}_{f}(M \bar{g})$ being $\mathbb{Q}$-dependent on $G(M) \bar{g}$; the remaining cases say that $\operatorname{acl}_{f}(M \bar{g})$ is self-sufficiently embedded.

Clearly we can ensure that $\Phi$ contains $L^{P}$-formulas expressing the first group of conditions. For the second, we use Proposition 3.2 of [9]. Suppose $V(\bar{y}, \bar{z})$ is a variety over $\mathbb{Q}$, with the length of $\bar{y}$ being 
$k=n+2 s+1$. For an appropriate tuple of parameters $b$, we denote by $V(b)$ the variety in $k$-space defined by $V(\bar{y}, b)$. There is a finite set of proper basic tori $T_{1}, \ldots, T_{t}$, depending only on $V$, with the property that for any torus $T$ and any $b$, any irreducible component of $V(b) \cap T$ of dimension greater than $\operatorname{dim} V(b)+\operatorname{dim} T-k$ (an atypical component) is contained in a coset of one of the $T_{i}$.

Then in $\Phi$ we also take, for every such variety $V$ over $\mathbb{Q}$ a formula (with appropriate $r, t, T_{i}$ ):

$$
(\forall \bar{e})\left(\forall b \in P^{r}\right)
$$

$\left({ }^{\prime} V(b)\right.$ irreducible of dimension $\leq n+s^{\prime} \wedge((\bar{x}, \bar{e}) \in V(b)) \wedge G(\bar{e})$

$$
\left.\longrightarrow(\bar{x}, \bar{e}) \in \bigcup_{i \leq t}\left(P^{\times}\right)^{n+2 s+1} T_{i}\right) .
$$

To prove the claim we show that for any pair $M_{1} \preceq N_{1}$ of models of $T_{G}$ and $n$-tuple $\bar{g}$ in $N_{1}$, we have $\left(M_{1}, N_{1}\right)=\Phi(\bar{g})$ iff $\bar{g}$ is a Morley $n$-sequence of the green generic over $P\left(N_{1}\right)=M_{1}$.

First, suppose $\left(M_{1}, N_{1}\right) \models \Phi(\bar{g})$. So the $g_{i}$ are algebraically independent over $M_{1}$ and we need to show that the second bullet point above holds. Take $e_{1}, \ldots, e_{2 s+1}$ as there and let $V(b)$ be an irreducible variety over $M_{1}$ with $\bar{g} \bar{e}$ as generic point. So $V$ is a variety over $\mathbb{Q}$ and $b$ is a tuple of parameters in $M_{1}$, and $V(b)$ is of dimension at most $n+s$. By the appropriate element of $\Phi$, the elements of $\bar{g} \bar{e}$ satisfy $g_{1}^{a_{1}} \ldots g_{n}^{a_{n}} e_{1}^{b_{1}} \ldots e_{2 s+1}^{b_{2 s+1}}=c \in M_{1}$, for some integers $a_{i}, b_{j}$ not all zero. Clearly $c \in G\left(M_{1}\right)$, so this gives what we want.

Conversely suppose $\bar{g}$ is a Morley $n$-sequence of the green generic over $P\left(N_{1}\right)=M_{1}$. We need to show that it satisfies the formulas in $\Phi$. So take $V, b$ and $\bar{e}$ as in the displayed formula with $\bar{g} \bar{e} \in V(b)$. Let $T$ be the minimum torus of $\bar{g} \bar{e}$ over $M_{1}$ and $W$ the connected component of $V(b) \cap T$ containing $\bar{g} \bar{e}$. We claim this is atypical. Indeed, $\operatorname{dim} W=n+\operatorname{trdeg}\left(\bar{e} / M_{1} \bar{g}\right)$ and $\operatorname{dim} T=n+\mathrm{rk}_{\mathbb{Q}}\left(\bar{e} / M_{1} \bar{g}\right)\left(\right.$ where $\mathrm{rk}_{\mathbb{Q}}$ denotes multiplicative rank). As $\operatorname{acl}_{f}\left(M_{1} \bar{g}\right) \leq N_{1}$ we have

$$
2 \operatorname{trdeg}\left(\bar{e} / M_{1} \bar{g}\right) \geq \operatorname{rk}_{\mathbb{Q}}\left(\bar{e} / M_{1} \bar{g}\right) .
$$

Now, using these equations and the fact that $\operatorname{dim} V(b) \leq n+s$, we have that $\operatorname{dim} V(b)+\operatorname{dim} T-(n+2 s+1)<\operatorname{dim} W$ if $2 \operatorname{trdeg}\left(\bar{e} / M_{1} \bar{g}\right)-$ $\operatorname{rk}_{\mathbb{Q}}\left(\bar{e} / M_{1} \bar{g}\right)>\operatorname{trdeg}\left(\bar{e} / M_{1} \bar{g}\right)-s-1$. But the left hand side here is $\geq 0$, whereas the right hand side is negative, as we are supposing $\operatorname{trdeg}\left(\bar{e} / M_{1} \bar{g}\right) \leq s$. So $W$ is an atypical component of the intersection $V(b) \cap T$, and is therefore contained in a coset of one of the basic tori $T_{i}$. Both $V(b)$ and $T$ are defined over $M_{1}$, so $W$ is defined over $M_{1}$. Therefore the coset representative can be taken in $M_{1}$ : hence $\bar{g}$ satisfies the required formula in $\Phi$.

$(\square$ Claim 1)

Claim 2. In Case 2, we may take $c$ in $N$. 
Indeed, take $n>d(A / M)$, and $g_{1}, \ldots, g_{n} \in N$ as in Claim 1. Then for some $i \leq n$ we have $d\left(g_{i} / A M\right)=1$. But then $\operatorname{tp}\left(g_{i} / M_{1}\right)=$ $\operatorname{tp}\left(c / M_{1}\right)$, as required. ( $\square$ Claim 2)

Claim 3. In Case 1, we may take $c \in N$.

Again, take $n>d(A / M)$ but this time, consider $g_{1}, \ldots, g_{2 n} \in N$ as in Claim 1. Let $b_{i}=g_{i}+g_{2 i}$, for $i=1, \ldots, n$. Then one checks that these are white generics, independent over $M$. The same argument as in Claim 1 then shows that we can assume that $d\left(b_{1} / M A\right)=2$, so we can take $c=b_{1}$. ( $\square$ Claim 3)

We now deal with Case 3. There exists a tuple $\bar{c}=\left(c_{1}, \ldots, c_{2 n}\right)$ of elements of $M_{2}$ such that $c_{1}, \ldots, c_{n}$ are a transcendence basis of $M_{2}$ over $M_{1}$, and $c_{1}, \ldots, c_{2 n}$ is a $\mathbb{Q}$-basis for $G\left(M_{2}\right)$ over $G\left(M_{1}\right)$. It is enough to show that the type of $\bar{c}$ over $M_{1}$ is realised in $N$.

Let $V(b, \bar{y})$ be the variety over $M_{1}$ which has $\bar{c}$ as generic point. So $V(\bar{x}, \bar{y})$ is over $\mathbb{Q}$, and $b$ is a tuple of parameters in $M_{1}$.

Claim 4. There is a formula $\psi(\bar{x})$ in the field language such that $N \models$ $\psi(b)$ and if $\psi(f)$ holds (in an algebraically closed field of characteristic zero), then:

- $V(f, \bar{y})$ is irreducible and $\operatorname{dim} V(f, \bar{y})=n$

- the generic of $V(f, \bar{y})$ is multiplicatively independent over $\operatorname{acl}_{f}(f)$

- if $\bar{g}$ is a generic point of $V(f, \bar{y})$ and we colour its coordinates (and a torsion-free divisible multiplicative group generated by them) green, then $\operatorname{acl}_{f}(f) \leq \operatorname{acl}_{f}(f \bar{g})$.

The proof of this is in Poizat's paper ([9], from bottom of page 1674). $(\square$ Claim 4)

There is a set $\Psi(\bar{x}, \bar{y})$ of $L^{P}$-formulas which says:

- $V(\bar{x}, \bar{y})$ and $G(\bar{y})$

- $\operatorname{trdeg}(\bar{y} / P \bar{x})=n$

- $\bar{y}$ is multiplicatively independent over $P \bar{x}$

Claim 5. For each $\phi(\bar{x}, \bar{y}) \in \Psi$, the closed formula

$$
\forall \bar{x}(\psi(\bar{x}) \rightarrow(\exists \bar{y}) \phi(\bar{x}, \bar{y}))
$$

is in $T^{P}$ (the theory of belles paires).

Let $M^{\prime} \preceq N^{\prime}$ be a belle paire and $f$ a tuple in $N^{\prime}$ satisfying $\psi$. Let $A=\operatorname{acl}_{f}(f)$. Take $B=\operatorname{acl}_{f}(f \bar{g})$ as in the third bullet point in Claim 4. By the relative $\omega$-saturation in the belle paire, the free amalgam of $B$ and $\mathrm{cl}_{N^{\prime}}\left(M^{\prime} f\right)$ embeds into $N$ over $\mathrm{cl}_{N^{\prime}}\left(M^{\prime} f\right)$. Let $\bar{g}^{\prime}$ be the image of $\bar{g}$ under this embedding. Then $\left(M^{\prime}, N^{\prime}\right)=\Psi\left(f, \bar{g}^{\prime}\right)$. ( $\square$ Claim 5)

We can now finish off the proof in Case 3. Recall that $(M, N)$ is an $\omega^{+}$-saturated model of $T_{G}^{P}$. Because of the tuple $\bar{c}$, we have $N_{1} \models \psi(b)$. By Claims 4 and 5 and the $\omega^{+}$-saturation, there is therefore a tuple $\bar{c}^{\prime}$ in $N$ with $(M, N) \models \Psi\left(b, \bar{c}^{\prime}\right)$. As $M_{1} \leq N_{1}$ and $\delta\left(\operatorname{acl}_{f}\left(M_{1} \bar{c}^{\prime}\right) / M_{1}\right)=0$, we have $\operatorname{acl}_{f}\left(M_{1} \bar{c}^{\prime}\right) \leq N_{1}$. From this and $\Psi$, we obtain $\operatorname{tp}_{N_{1}}\left(\bar{c}^{\prime} / M_{1}\right)=$ $\operatorname{tp}_{N_{1}}\left(\bar{c} / M_{1}\right)$, as required. ( $\square$ Theorem) 
We remark that Martin Hils pointed out to us that Theorem 3.3 follows from a result of his [2]: the theory of $T_{G}$ (in a language expanded by definitions so that it is model complete) together with an automorphism has a model companion.

3.3. Variations on green points. In [11], Zilber proves that, under the assumption of Schanuel's conjecture, the field of complex numbers expanded by a predicate for the multiplicative subgroup

$$
\exp ((1+\mathrm{i}) \mathbb{R}+\mathbb{Q})
$$

is a model of Poizat's $T_{G}$. For the purposes of [13], it is more natural to consider $\mathbb{C}$ expanded by predicates for slightly different multiplicative subgroups.

Example 3.4. (See Section 3.5 of [13].) Consider $\alpha, \beta \in \mathbb{C}^{\times}$linearly independent over $\mathbb{R}$, and let $i_{a}, i_{b} \in \mathbb{R}$ be such that $\mathrm{i}=i_{a} \alpha+i_{b} \beta$. Let $h \in \mathbb{R}$ be such that $1,2 \pi i_{a}, 2 \pi i_{a} h$ are linearly independent over $\mathbb{Q}$ and let $N \in \mathbb{N}$. Define

$$
G_{0}=\exp \left(\frac{2 \pi \mathrm{i}}{h N} \mathbb{Z}+\frac{\alpha}{h} \mathbb{Z}+\beta \mathbb{R}\right) .
$$

There is a theory $\tilde{T}_{G}$ axiomatised in the same way as Poizat's $T_{G}$, but where the subgroup of 'green' points is elementarily equivalent to $\mathbb{Z}^{2}$, rather than $\mathbb{Q}$. The theory $\tilde{T}_{G}$ is superstable of $U$-rank $\omega .2$ and assuming Schanuel's conjecture, $\tilde{T}_{G}=T h\left(\mathbb{C}, G_{0}\right)$ (see Proposition 3.5 of [13]). (One caveat here is that the class of coloured algebraically closed fields $(A, G(A))$ of characteristic zero with $G(A) \equiv \mathbb{Z}^{2}$ satisfies the amalgamation property but not the joint embedding property. So one should really work with the subclass of coloured fields containing a fixed $\left(A_{0}, G\left(A_{0}\right)\right)$, say with $\delta\left(A_{0}\right)=0$, and include constants for generators of $G\left(A_{0}\right)$ in the language.)

In the notation of Definition 1.4, we expand $\left(\mathbb{C}, G_{0}\right)$ to an $L_{0}$-structure by setting $\Gamma=\langle\epsilon\rangle ; G=G_{0} \cdot \Gamma ; G^{+}=\exp ((2 \pi \mathrm{i} / h N) \mathbb{Z}+(\alpha / h N) \mathbb{Z}+$ $\beta \mathbb{R}) . \Gamma$ and $\chi(a)=\epsilon$, where $\epsilon$ is a primitive $N$-th root of 1 , and $a=\exp (\alpha / h N)$. Note that $G$ is of index $N$ in $G^{+}$and so all of these are definable in $\left(\mathbb{C}, G_{0}, a\right)$. Thus (assuming Schanuel's conjecture) we obtain a structure whose theory $T_{0}$ is superstable of rank $\omega .2$. The proof of nfcp for $T_{G}$ given in Section 3.2 also works for $\tilde{T}_{G}$ with little modification. So Theorem 1.5 applies, and as in Theorem 3.2 we have:

Theorem 3.5. With the above notation, and that of Definition 1.4:

(i) $T_{0}$ is superstable of Lascar rank $\omega .2$ and has $n f c p$.

(ii) $T_{A}$ has a model completion $T_{A}^{*}$ and all completions of this are supersimple of $S U$-rank $\omega .2$.

In Section 3.5 of [13] Zilber gives an explicit construction of an angular function $\operatorname{ang}_{N}$ with the above data and asks whether the theory of the complex numbers expanded by $\operatorname{ang}_{N}$ is supersimple (assuming 
Schanuel's conjecture). In the light of the above result, it appears reasonable to ask whether it is a model of $T_{A}^{*}$.

Example 3.6. In Section 3.3 of [13] Zilber works with an angular function ang having the following data: $\Gamma=\exp (2 \pi \mathrm{i} h \mathbb{Z}) ; G=\exp (2 \pi \mathrm{i} h \mathbb{Z}+$ $\beta \mathbb{R}) ; G^{+}=\exp (2 \pi \mathrm{i} h \mathbb{Z}+\alpha \mathbb{Z}+\beta \mathbb{R})$ and $\chi(\exp (\alpha))=\exp (2 \pi \mathrm{i} h)$, where $\alpha, \beta$ and $h$ are as in Example 3.4. Again, Schanuel's conjecture implies that $T h(\mathbb{C} ; G, \Gamma)$ can be axiomatized in a similar way to Poizat's $T_{G}$ (the appropriate predimension to use is $\delta(A)=2 \cdot \operatorname{trdeg}(A)-\mathrm{rk}_{\mathbb{Q}}(\mathcal{G}(A))-$ $\operatorname{rk}_{\mathbb{Q}}(\Gamma(A))$ ), and is superstable of Lascar rank $\omega .2$ (see Proposition 2 of Section 3.3 in [13]). However, as $\Gamma$ is infinite, the group $G^{+}$and the homomorphism $\chi$ are not definable in this, so we require a slightly different approach.

Let $L_{0}^{\prime}$ be a language consisting of the language of rings together with unary predicates $\mathcal{G}, \Gamma, \Gamma^{+}$and unary functions $\chi, \chi^{-1}$. Consider the class of $L_{0}^{\prime}$-structures consisting of an algebraically closed field $A$ of characteristic zero, and multiplicative subgroups $\mathcal{G}(A), \Gamma(A), \Gamma^{+}(A)$ with the properties that

- $\Gamma(A)$ is a pure subgroup of $\mathcal{G}(A)$ and $\mathcal{G}(A) \cap \Gamma^{+}(A)=1$;

- the groups $\mathcal{G}(A)$ and $\Gamma(A)$ are elementarily equivalent to $\mathbb{Z}$;

- $\chi: \Gamma^{+}(A) \rightarrow \Gamma(A)$ and $\chi^{-1}: \Gamma(A) \rightarrow \Gamma^{+}(A)$ are mutually inverse group isomorphisms;

- the predimension inequality $\delta \geq 0$ holds,

where $\delta(A)=2 \cdot \operatorname{trdeg}(A)-\operatorname{rk}_{\mathbb{Q}}(\mathcal{G}(A))-3 \cdot \operatorname{rk}_{\mathbb{Q}}(\Gamma(A))$. Note that the intention is that we can take (definably) $\mathcal{G}^{+}$to be the direct product $\mathcal{G} . \Gamma^{+}$and $\chi$ extends to $\mathcal{G}^{+}$by projection to the second factor. As with Poizat's $T_{G}$ one can construct a complete $L_{0}^{\prime}$-theory $\hat{T}_{G}$ whose $\omega$-saturated models are the 'rich' structures in the class; moreover $\hat{T}_{G}$ is superstable of Lascar rank $\omega .2, \mathcal{G}$ is of rank $\omega$ and $\Gamma$ of rank 1 . (As in 3.4 we should really work over a fixed coloured field.) This is similar to the construction in [12] and as there, it is expected that $\left(\mathbb{C} ; \Gamma, G, G^{+}, \chi\right)$ as given above is a model of $\hat{T}_{G}$ (as always, assuming Schanuel's conjecture), though we have not checked the details.

The proof of Theorem 3.3 can easily be adapted to show that $\hat{T}_{G}$ has nfcp. Thus, as in Theorem 1.5, the theory $\left(\hat{T}_{G}\right)_{A}$ of models of $\hat{T}_{G}$ (with the language expanded to get model completemenss) with an angular function has a model companion $\left(\hat{T}_{G}\right)_{A}^{*}$. Note that by Remark 2.6, this is not simple.

The above provides one possible approach to Problems 1 and 2 of Section 3.3 of [13] and it is of course of interest to check whether Zilber's explicit angular function ang with the above data gives a model of $\left(\hat{T}_{G}\right)_{A}^{*}$. 


\section{A probability measure}

In this section we describe (- under certain hypotheses on $T_{0}$ ) how to put a probability measure on the definable subsets of $F$ in models of $T_{A}^{*}$. In particular, this gives one possible answer to the Problem in 2.4 of [13].

We continue to use the notation of Definition 1.4 and assume that $T_{0}$ is a superstable $L_{0}$-theory with nfcp and $\Gamma$ is finite; we also assume that $T_{0}$ has quantifier elimination (as usual, this can be achieved by expanding the language). As we are working with superstable fields, there is a unique 1-type of maximal rank (- the generic type) over any algebraically closed subset of a model of $T_{0}$. We will assume that whenever $C$ is algebraically closed (in a model of $T_{0}$ ) and $e$ is generic over $C$, then $\mathcal{G}^{+}(\operatorname{acl}(C e))=\mathcal{G}^{+}(C)$. This is the case in the examples of interest in Section 3.

Theorem 1.5 applies, and we fix a completion of $T_{A}^{*}$, which we will also denote by $T_{A}^{*}$. Results in [5] imply that this is determined by the values of the angular function on $\operatorname{acl}(\emptyset)$; it is supersimple; algebraic closure in the $T_{A}^{*}$ and $T_{0}$ senses are the same, and in a model ( $F$; ang) of $T_{A}^{*}$ a generic type $\operatorname{tp}(e / C)$ is determined by the restriction of the angular function ang to $\operatorname{acl}(\mathrm{Ce})$. (Of course, the results in [5] apply to $T_{s}^{*}$, and we obtain the corresponding results about $T_{A}^{*}$ as in the proof of Theorem 1.5.)

Work in a big model ( $F$; ang) of $T_{A}^{*}$ and let $C \subset F$ be algebraically closed. Let $\mathcal{X}$ be the set of generic types over $C$, equipped with the Stone space topology. Note that $\mathcal{X}$ is closed in the Stone space of all $T_{A}^{*}$-types over $C$, as it consists of those types which extend the $T_{0}$ generic type over $C$. In particular, $\mathcal{X}$ is compact.

Fix some $e \in F$ which is generic over $C$ and let $B=\operatorname{acl}(C e)$. Consider $B$ just as an $L_{0}$-structure and let $\mathcal{Y}$ denote the set of all possible angular functions from $B$ to $\Gamma$ which extend the given ang $\mid C$. By the above remarks each element of $\mathcal{Y}$ determines a generic type over $C$, and two elements of $\mathcal{Y}$ give rise to the same generic type iff there is an $L_{0}$-automorphism of $B$ over $C e$ which sends one to the other. In summary:

Lemma 4.1. There is a surjective map $\Theta: \mathcal{Y} \rightarrow \mathcal{X}$ whose fibres are the $\operatorname{Aut}_{L_{0}}(B / C e)$-orbits on $\mathcal{Y}$.

Note that the quotient of any two elements of $\mathcal{Y}$ (as functions $B \rightarrow \Gamma$ ) is constant on $\mathcal{G}^{+}(B)$-cosets, and so is a function from $B / \mathcal{G}^{+}(B)$ to $\Gamma$ which is the identity on $C / \mathcal{G}^{+}(B)$. Conversely if we multiply any element of $\mathcal{Y}$ by such a function, we obtain another element of $\mathcal{Y}$. Thus we have a regular action of the group

$$
P=\Gamma^{\left(B / \mathcal{G}^{+}(B)\right) \backslash\left(C / \mathcal{G}^{+}(B)\right)}
$$

of such functions on $\mathcal{Y}$. 
Now, $P$ is a product of copies of the finite set $\Gamma$, so we can give it the product topology. We can regard $\Gamma$ as a uniform probablility space, and give $P$ the product measure $\kappa$ (of course, this is the Haar measure on $P$ ). By choosing an element $y_{0}$ of $\mathcal{Y}$ we obtain a bijection $\eta: P \rightarrow \mathcal{Y}$ (with $\eta(g)=g y_{0}$ ), and thereby can transfer the topology and measure on $P$ to $\mathcal{Y}$. The resulting topology and measure $\lambda$ on $\mathcal{Y}$ are independent of the choice of $y_{0}$ here.

Claim: the map $\Theta$ in the above lemma is continuous.

For $s \in \mathbb{N}$ and $\bar{a}=\left(a_{1}, \ldots, a_{s}\right) \in B^{s}$, let $\psi\left(x_{1}, \ldots, x_{s}, y\right)$ be an $L_{0}(C)$-formula such that $\psi(\bar{x}, e)$ isolates the $L_{0}$-type of $\bar{a}$ over $C e$. An $L_{A}(C)$-formula $\theta(y)$ of the form

$$
(\exists \bar{x})\left(\psi(\bar{x}, y) \wedge \bigwedge_{i=1}^{s}\left(\operatorname{ang}\left(x_{i}\right)=\gamma_{i}\right)\right)
$$

for $\gamma_{1}, \ldots, \gamma_{s} \in \Gamma$ will temporarily be called a basic formula. Then $p, q \in \mathcal{X}$ are equal iff they contain the same basic formulas, so the clopen sets determined by the basic formulas are a basis of the topology on $\mathcal{X}$.

Now, for any such basic $\theta(y)$ there is a finite $A \subseteq B$ such that, for $f, g \in \mathcal{Y}$, if $f, g$ have the same restriction to $A$, then $\theta(y) \in \Theta(f)$ iff $\theta(y) \in \Theta(g)$ (simply take $A$ to contain all $\operatorname{Aut}(B / C e)$-translates of the $\left.a_{i}\right)$. Continuity of $\Theta$ then follows.

Definition 4.2. If $D$ is a $C$-definable subset of $F$, let $[D]$ be the closed subset $\{p \in \mathcal{X}: D \in p\}$ of $\mathcal{X}$, and define

$$
\mu(D)=\lambda\left(\Theta^{-1}([D])\right) .
$$

This is a finitely-additive measure on the collection of $L_{A}(C)$-definable subsets of $F$. Of course, as $\lambda$ is a measure on the Borel sets of $\mathcal{Y}, \mu$ extends to a $\sigma$-additive measure on the collection of countable boolean combinations of $C$-definable sets.

Lemma 4.3. The measure $\mu$ does not depend on the choice of $C$.

Proof. Temporarily augment the notation by including a subscript to indicate dependency on $C$. We need to show that if $C^{\prime} \supseteq C$ is algebraically closed and $D$ is $C$-definable, then $\mu_{C}(D)=\mu_{C^{\prime}}(D)$.

Let $e \in F$ be generic over $C^{\prime}$ and $B^{\prime}=\operatorname{acl}\left(C^{\prime} e\right)$. Then $e$ is also generic over $C$, and we let $B=\operatorname{acl}(C e)$. Obviously $\mathcal{G}^{+}\left(B^{\prime}\right) \cap B=\mathcal{G}^{+}(B)$ so there is a natural injective function $\iota: B / \mathcal{G}^{+}(B) \rightarrow B^{\prime} / \mathcal{G}^{+}\left(B^{\prime}\right)$ (given by $x \mathcal{G}^{+}(B) \mapsto x G^{+}\left(B^{\prime}\right)$ ). By the assumption that $\mathcal{G}^{+}\left(B^{\prime}\right)=$ $\mathcal{G}^{+}\left(C^{\prime}\right)$, it follows that $\iota^{-1}\left(C^{\prime} / \mathcal{G}^{+}\left(B^{\prime}\right)\right)=C / \mathcal{G}^{+}(B)$. Thus $\iota$ induces a surjective map $\pi: P_{C^{\prime}} \rightarrow P_{C}$ (essentially by restriction), and of course this is a continuous group homomorphism.

Let $\eta_{C^{\prime}}: P_{C^{\prime}} \rightarrow \mathcal{Y}_{C^{\prime}}$ and $\eta_{C}: P_{C} \rightarrow \mathcal{Y}_{C}$ be the bijections obtained by taking $\eta_{C^{\prime}}(1)=\operatorname{ang} \mid C^{\prime}$ and $\eta_{C}(1)=\operatorname{ang} \mid C$. Restriction gives a continuous map $\rho: \mathcal{Y}_{C^{\prime}} \rightarrow \mathcal{Y}_{C}$ and the square 


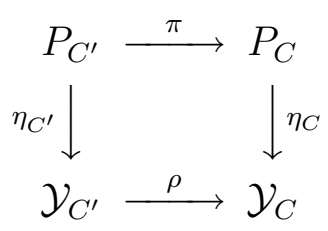

is commutative. In particular, $\rho$ is surjective.

Restriction also gives a continuous surjection $\tau: \mathcal{X}_{C^{\prime}} \rightarrow \mathcal{X}_{C}$, and the square:

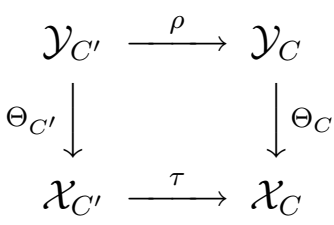

is commutative.

Note that if $D$ is $C$-definable, then $[D]_{C^{\prime}}=\tau^{-1}[D]_{C}$ (i.e. any generic type over $C$ containing $D$ extends to one over $C^{\prime}$ ). So we need to verify that if $Z \subseteq \mathcal{X}_{C}$ is closed then

$$
\lambda_{C^{\prime}}\left(\Theta_{C^{\prime}}^{-1} \tau^{-1}(Z)\right)=\lambda_{C}\left(\Theta_{C}^{-1}(Z)\right) .
$$

By commutativity of the square, this follows if we show that whenever $Y \subseteq \mathcal{Y}_{C}$ is closed (or even Borel), then

$$
\lambda_{C^{\prime}}\left(\rho^{-1}(Y)\right)=\lambda_{C}(Y) .
$$

By the first square and definition of $\lambda$, this follows if $\kappa_{C^{\prime}}\left(\pi^{-1}(X)\right)=$ $\kappa_{C}(X)$ for all closed (or Borel) $X \subseteq P_{C}$. But each side of this equation is a Haar measure on $P_{C}$ : hence the equality.

Example 4.4. The following example shows that the measure $\mu$ is not preserved under definable bijections.

Let $T_{0}$ be as in Example 3.4, $N \in \mathbb{N}$ not divisible by 2 and $\Gamma$ the $N$-th roots of unity, with $\epsilon$ a primitive $N$-th root. Let $T_{A}^{*}$ be as in Theorem 3.5. Consider the $L_{A}(\Gamma)$-definable sets:

$$
\begin{aligned}
& X=\left\{e \in F^{*}: \operatorname{ang}(e)=1, \operatorname{ang}\left(\left\{g: g^{2}=e\right\}\right)=\left\{\epsilon, \epsilon^{2}\right\}\right\} \\
& Y=\left\{g \in F^{*}: \operatorname{ang}(g)=\epsilon, \operatorname{ang}\left(g^{2}\right)=1, \operatorname{ang}(-g)=\epsilon^{2}\right\} .
\end{aligned}
$$

There is a definable bijection between these sets as the different values of the angular function allow one to select in a definable way a square root of each $e \in X$. On the other hand, $\mu(X)=2 / N^{3}$ whereas $\mu(Y)=$ $1 / N^{3}$. 


\section{REFERENCES}

[1] Enrique Casanovas and Martin Ziegler, 'Stable theories with a new predicate,' J. Symbolic Logic 66 (2001), 1127-1140.

[2] Martin Hils, Fusion Libre et Autres Constructions Génériques, Thèse, Université Paris 7, July 2006.

[3] Wilfrid Hodges, Model Theory, Cambridge University Press, Cambridge, 1993.

[4] Ehud Hrushovski and Boris Zilber, 'Zariski geometries,' J. American Math. Soc. 9 (1996), 1-56.

[5] Herwig Nübling, 'Adding Skolem functions to simple theories,' Arch. Math. Logic 43 (2004), 359-370.

[6] Anand Pillay, Geometric Stability Theory, Oxford Logic Guides 32, Clarendon Press, Oxford, 1996.

[7] Anand Pillay, 'Lang's conjecture and model theory', in Model Theory and Algebraic Geometry, E. Bouscaren (ed.), Springer LNM 1696, pp. 101-106, Springer, 1998/1999.

[8] Bruno Poizat, 'Paires de structures stables,' J. Symbolic Logic 48 (1983), 239249.

[9] Bruno Poizat, 'L'égalité au cube,' J. Symbolic Logic 66 (2001), 1647-1676.

[10] Peter M. Winkler, 'Model-completeness and Skolem expansions,' in Model Theory and Algebra - A memorial tribute to Abraham Robinson, D. H. Saracino and V. B. Weisspfenning (eds.), Lecture Notes in Mathematics, 498, SpringerVerlag, 1975, pp. 408-463.

[11] Boris Zilber, 'Bi-coloured fields on the complex numbers', J. Symbolic Logic 69 (2004), 1171-1186.

[12] Boris Zilber, 'A remarkable pseudo-analytic structure', webpage, Oxford, August 2003.

[13] Boris Zilber, 'Non-commutative geometry and new stable structures', Preprint, Oxford, November 2005.

School of Mathematics, University of East Anglia, Norwich NR4 7TJ, ENGLAND

E-mail address: d.evans@uea.ac.uk 